\title{
Tacrolimus Therapeutic Drug Monitoring in Kidney Transplant Patients Before and After Pharmacist Post-transplant Consults
}

\author{
Gabriella Massoglia, PharmD ${ }^{1}$; Murraysha Ramnarine, PharmD²; Michael J. Schuh, PharmD, MBA, FAPhA \\ ${ }^{1}$ Mayo Clinic Florida; ${ }^{2}$ University of Florida College of Pharmacy
}

\begin{abstract}
Objectives: This quality improvement project sought to determine if clinical pharmacist office visits, or consults, had an impact on therapeutic tacrolimus levels in patients after kidney transplant through analysis of tacrolimus serum concentrations before versus after a pharmacist visit.

Methods: A retrospective chart review was conducted for all patients that attended a clinical pharmacist office visit, also known as a consult, after kidney transplantation for a four month period. Pharmacists during the post-transplant consult reviewed adherence but also educated patients in detail about timing and logging of tacrolimus dosing, dosing with regard to labs to assure trough dosing, dosing with or without food in the stomach, what to do with a dose is taken late or if a dose is missed and foods, herbal supplements to avoid that may inhibit CYP3A4 to elevate tacrolimus levels.

Results: After the post-transplant visit with a clinical pharmacist there was a $46 \%$ increase in the number of tacrolimus trough levels in therapeutic range versus before the pharmacist visit. Sixty-four percent of kidney transplant patients experienced an increase in the number of therapeutic drug levels in range after the pharmacist visit versus before the pharmacist visit.

Conclusions: An increase in the quantity of therapeutic tacrolimus trough levels and the number of patients with therapeutic drug levels in range was observed after patients received pharmacist provided medication education post-transplant with emphasis on proper immunosuppressant medication administration, adherence and potential drug and food interactions. This finding demonstrates the importance of pharmacist clinical services in producing improved therapeutic drug levels in post-transplant kidney patients.
\end{abstract}

\section{Introduction}

Nonadherence to immunosuppressant therapy is the leading cause of preventable graft failure in post-transplant patients. ${ }^{1,2}$ Other factors that contribute to graft loss include calcineurin inhibitor-induced nephrotoxicity and lack of patient understanding regarding the prescribed post-transplant medication regimen., ${ }^{3,4}$ Addressing these risk factors is imperative for graft preservation and patient survival. The field of transplant medicine has grown tremendously since 1954 when the first successful human organ transplant was performed. ${ }^{5}$ In the United States, a total of 36,528 transplants were performed in 2018, of which 21,167 were kidney transplants. ${ }^{6,7}$ Despite advances in knowledge and medical technology, graft rejection resulting from nonadherence and resultant immunosuppressant therapeutic values being out of range continues to be a complication for patients after transplant. Medication nonadherence, particularly to immunosuppressant agents, has been linked to a 7-fold increase in graft rejection. ${ }^{4}$ Pinsky and colleagues found that patients who were non-adherent to immunosuppressant medications for greater than $4.9 \%$ of days were at a $60 \%$ increased risk of graft rejection. ${ }^{8}$ Pharmacist consultations

\section{Corresponding Author:}

Michael J. Schuh, PharmD, MBA, FAPhA

Mayo Clinic Florida

4500 San Pablo Rd, Jacksonville, FL 32224

Phone904-953-2673; Email: schuh.michael@mayo.edu during the early post-transplant period was shown in this project to ensure positive patient outcomes through medication education about proper immunosuppressant medication administration, monitoring of drug serum concentration levels and potential drug and food interactions. Transplant ambulatory pharmacists at this institution do not have prescribing or lab ordering privileges so recommendations are made with regard to identified medication related problems (MRPs) and other emergent, actionable issues are immediately reported to the patients transplant nurse coordinator or physician to be resolved.

\section{Background}

As medication experts, pharmacists play a fundamental role on the transplant team. At Mayo Clinic Florida, pharmacists are involved throughout the transplant process. Pre-transplant activities include collaborating as part of a multidisciplinary healthcare team during candidate selection committee meetings, conducting face-to-face pre-transplant assessment visits or consults, and instructing weekly medication education classes that focus on the post-transplant regimen and side effect management. By participating in these activities, pharmacists help reduce the risk of graft rejection by addressing pharmacological, nonpharmacological factors and potential barriers to the best therapeutic outcomes. Pharmacological factors such as dosage adjustments needed for dialysis or renal/hepatic impairment are considered during the one-on-one pre-transplant consults. In addition to optimization of patients' current drug therapy regimen to avoid drug-drug and drug-herbal interactions with the anticipated post-transplant medication regimen, barriers to adherence are 
also evaluated and addressed. Non-pharmacologic factors are also taken into consideration to identify patients that may be at an increased risk of organ rejection due to nonadherent behaviors and socioeconomic or communication barriers posttransplant. To resolve these issues, pharmacists create patient specific plans to address the barriers that could potentially preclude transplantation.

In order to become an eligible candidate to be listed for transplant, patients must undergo consults with several specialties including psychiatry, nutrition, surgery and pharmacy. Historically, the pharmacist post-transplant consults at the institution were led by pharmacists with specialized training in solid-organ transplant even though they are not specifically required by CMS (Centers for Medicare and Medicaid Services). However, in March 2015, pharmacist consults pre-transplant also became a mandatory part of the Mayo Clinic protocol per CMS guidelines. Since pre-transplant pharmacist visits were now CMS required but not posttransplant visits, post-transplant pharmacist consults were reviewed as a quality project to determine if they improved patient outcomes. Results of the project would help determine if post-transplant consults continued.

Post-transplant pharmacist consults occur face-to-face, preferably 3-7 days after transplant. The goal of these appointments is to ensure graft preservation through a patient centered care approach. This is achieved via in depth counseling session where the pharmacist both assesses and educates each patient. The patient post-transplant assessment consists of reviewing the medication fill/refill history, review of the logging of each medication dose and daily vitals in the patient's post-transplant binder and review of the patient's electronic medical record. The patient-managed binder is an organizational tool to record vitals, hydration status, weight changes, and medication administration times and doses. The pharmacist assesses patient adherence through review of the binder, as well as makes recommendations for therapy changes to improve tolerability, adherence, and assist with achieving target serum concentrations. Key patient education during the session consists of proper timing and frequency of dose administration, the effect of meals or empty stomach on drug levels and efficacy, what to do with missed doses, how to properly manage side effects, how to avoid drugdrug/food/herbal interactions and the potential consequences of nonadherence. Reinforcement of the value of adherence tools such as alarms and pill boxes is another beneficial part of the consult. The pharmacist concludes each session by addressing any patient knowledge deficiencies and addressing any questions, concerns or identified MRPs. Any recommendations to the provider and nurse coordinator are then made in the pharmacist clinical note and any acute, emergent issues are immediately addressed by contacting the nurse coordinator and/or attending physician.

\section{Materials and Methods}

To determine if pharmacist assessment and education positively impacted patients' tacrolimus levels in the posttransplant stage, a quality improvement project and retrospective chart review was performed. The institution has performed post-transplant pharmacist consults since inception of each of its solid organ transplant programs. Since posttransplant pharmacist consults are not required by CMS, a quality project was performed to determine if the pharmacist labor invested on these consults resulted in better patient outcomes via an increased percentage of in range therapeutic tacrolimus levels or a higher number of patients with levels in range. If patient outcomes were not improved, post-transplant consults would be stopped. This project demonstrates the added value of pharmacist led post-transplant consults since improved patient outcomes were achieved. This project was approved as a quality project by the Institutional Review Board prior to initiation. Data was collected from the institution's electronic health record from March 2, 2015 to March 30, 2016. Extracted data included the patient's age, sex, transplant date, consult date, number of days elapsed since discharge on date of consult, goal tacrolimus levels, number of sub and supratherapeutic tacrolimus trough serum concentrations before and after pharmacist post-transplant consult, and the number of therapeutic tacrolimus trough serum concentrations before and after pharmacist post-transplant consult. The transplant notes were utilized to determine the patient's immunosuppressant therapy goals, with all notes being reviewed to ensure that these goal levels did not change over time due to toxicity or other reasons.

Key educational points covered during the pharmacist consult were timing and logging of tacrolimus dosing, dosing with regard to labs to assure trough dosing, dosing with or without food in the stomach, what to do with a dose is taken late or if a dose is missed and foods, herbal supplements to avoid that may inhibit CYP3A4 to elevate tacrolimus levels.

Cases where the physician chose to deviate from the protocol were noted and were analyzed accordingly. Patients who received dual transplants, prior transplants, or had orders to discontinue tacrolimus therapy were excluded from analysis. Tacrolimus trough levels were assessed up to 4 months after transplant. The number of supra and sub-therapeutic tacrolimus levels before and after pharmacist post-transplant consult were counted and recorded, being cognizant of the fact that goals change after 1 month of therapy. The percentage of tacrolimus trough levels in therapeutic range prior to and after pharmacist consult were compared to determine if improvement occurred, defined as more tacrolimus therapeutic levels in range or more patients with therapeutic blood levels. 


\section{Results}

Data collected for analysis included the patient's age, sex, transplant date, pharmacist visit date, number of days elapsed since discharge from the date of the visit, goal tacrolimus level, number of sub and supratherapeutic tacrolimus trough serum concentrations before and after pharmacist post-transplant visit, and the number of therapeutic tacrolimus trough serum concentrations before and after pharmacist post-transplant visits.

Inclusion criteria was any patient who followed a normal transplant therapy course defined as no deaths, extra hospitalizations or stay longer than the one month posttransplant clinic follow-up, then discharge to home. Exclusions were re-transplantation or prior transplant of another organ, dual organ transplant, or if cyclosporine was ever used instead of tacrolimus. Total patients reviewed were 127 with 22 exclusions, leaving a sample size of 105. Average age was 50.6 years old, 45 of 105 patients being female, 60 male.

Target tacrolimus trough levels defined therapeutically as inrange were $8-10 \mathrm{ng} / \mathrm{mL}$ in the first 30 days post-transplant then 6-8 $\mathrm{ng} / \mathrm{mL}$ day 31-60 post-transplant and thereafter. The average number of labs taken pre-pharmacist consult was 7.86, post consult 12.33. The average number of days seen postdischarge was 13.2. After kidney transplant, patients had an average of $24 \%$ tacrolimus trough levels therapeutic before a pharmacist post-transplant consult; after pharmacist posttransplant consult, an average of $35 \%$ of tacrolimus trough levels were therapeutic (Figure I). This shows a $45.8 \%$ improvement in the number of therapeutic tacrolimus trough levels after the pharmacist consult. Sixty seven out of 105 (63.8\%) kidney transplant patients had an increased number of therapeutic tacrolimus trough levels after the pharmacist consult. Therefore, the number of patients with improved therapeutic levels increased along with the overall number of therapeutic levels in the studied population, indicating improvement could not just be attributed to a few patients with many in range therapeutic levels.

Patients post-transplant are tightly monitored by the posttransplant nurse coordinator with three times weekly tacrolimus levels taken with the number of levels decreasing if the patient remains in therapeutic range as the patient progresses in the post-transplant clinic. Because the patients are closely monitored and required to keep a monitored, detailed log book on dosing throughout their post-transplant clinic period, they are very adherent as a group with few exceptions. Therefore resultant higher therapeutic tacrolimus levels could be more attributed to pharmacist education factors than adherence problems since adherence is an intensely monitored factor throughout the transplantation process.

\section{Discussion}

Based on the findings of this project, pharmacist consults in the post-transplant period had a positive impact on the number of in range therapeutic tacrolimus trough levels and number of patients receiving in range trough levels after kidney transplant.

The preferred time post-transplant of a pharmacist seeing patients was exceeded on average by 7-10 days. This could be attributed to the volume of appointments each patient has daily due to frequent labs being drawn and many specialist and coordinator visits. Because post-transplant pharmacist consults are not CMS required, they are also triaged below CMS required and protocol driven appointments. This may have contributed to appointments being further out than desired.

Because visits were scheduled further out from discharge than preferred (13 days versus 3-7 days), this may have actually increased the number of in range therapeutic drug level results in the project pre-pharmacist consult since patients and caregivers are expected to be more knowledgeable and proficient managing the complex medication administration regimen with increasing time of nurse coordinator and physician contact via appointments or phone contact. Patients are least knowledgeable and are less likely to be in a medication administration routine when the patient is very early in recovery post-discharge. Earlier in the post-transplant process (3-7 days) there may have been fewer lab draws and those fewer labs more likely would have been out of range as a percentage pre-consult, resulting in skewed but more favorable outcome data as to more in range tacrolimus levels post pharmacist consult as a result. Therefore, seeing the patient later in the post-transplant recovery process may have assisted in preventing the skewing of the data to an even higher percentage difference in drug levels in range pre versus post periods around the pharmacist visit.

Pharmacist intervention during this critical period is essential in helping ensure graft preservation through monitoring and patient education, specifically regarding medication administration with special attention to presence of food, what to do when a diose is missed or late or consumption of foods or other agents that could affect tacrolimus levels. Implementing a pharmacist service to conduct post-transplant consults effectively helps reduce toxicity or graft rejection risk factors through the education, monitoring, and positive reinforcement. Pharmacists can also provide monitoring and dosage recommendations to achieve therapeutic drug levels and prevent calcineurin inhibitor-induced nephrotoxicity. 9,10

It was frequently discovered in the post-transplant pharmacist visit patients were very adherent with taking the tacrolimus dose twice daily, every 12 hours, but were not aware of the difference the amount of food on their stomach made on their therapeutic drug levels. Similarly, some did not know or didn't remember from previous education the effect CYP3A4 inhibiting foods or herbals had on tacrolimus levels or what to 
do in the case of a late or missed dose of tacrolimus. These may have been key reasons why pharmacist supplemental education made a difference in tacrolimus therapeutic blood levels.

\section{Limitations}

The retrospective nature of this quality improvement project creates several limitations in this study. Retrospective analysis is unable to account for variables or control confounding factors. Secondly, although there is a general template for pharmacists to use during post-transplant consults, no specific guidelines and training were set forth to create a standardized patient experience. Therefore, differences in delivery may vary based on pharmacist, which could lead to varying patient results. Furthermore, the use of serum concentration variability as a measure of adherence or education does not account for the multi-faceted medication consumption process and without including adverse effects and rejection rates, tacrolimus therapeutic levels could be considered a surrogate endpoint. Differences in pharmacokinetics due to changing renal function, metabolism, and drug-drug interactions cannot be accounted for with this method. Although this study provides positive results in terms of pharmacist impact on patient care in the post-transplant setting, future studies which use a prospective, controlled approach are needed to further validate our findings.

\section{Conclusion}

Non-adherence, or deviation from the prescribed posttransplant immunosuppressant regimen, is a leading cause of preventable graft failure, contributing to late acute rejection episodes and graft losses. ${ }^{1,2}$ Pharmacists can help detect and intervene upon nonadherence through individualized face-toface consults with patients after kidney transplant to ensure that patients understand the importance of their regimen and appropriate administration instructions. ${ }^{11,12}$ In this project, consulting with a pharmacist post-transplant increased the percentage of in range tacrolimus therapeutic trough levels and the number of patients experiencing tacrolimus therapeutic levels in range, even though this patient sample population was considered adherent to their medication regimen.

Conflict of interest: None of the authors received any financial interest, direct or indirect, related to the achievement of this work.

\section{References}

1. Nevins TE, Nickerson PW, Dew MA. Understanding Medication Nonadherence after Kidney Transplant. J Am Soc Nephrol. 2017;28(8):2290-2301. doi:10.1681/ASN.2017020216

2. Marie A. Chisholm, Charles E. Lance, Gail M. Williamson, Laura L. Mulloy, Development and validation of an immunosuppressant therapy adherence barrier instrument, Nephrology Dialysis Transplantation, Volume 20 , Issue 1 , January 2005, Pages 181188, https://doi.org/10.1093/ndt/gfh576

3. El-Husseini A, Aghil A, Ramirez J et al. Outcome of kidney transplant in primary, repeat, and kidney-after-nonrenal solid-organ transplantation: 15 -year analysis of recent UNOS database. Clin Transplant. 2017;31(11):e13108.

4. Jamieson N, Hanson C, Josephson M et al. Motivations, Challenges, and Attitudes to Self-management in Kidney Transplant Recipients: A Systematic Review of Qualitative Studies. American Journal of Kidney Diseases. 2016;67(3):461-478.

5. History - UNOS. UNOS. https://unos.org/transplant/history/. Published 2019.

6. Transplant trends - UNOS. UNOS. https://unos.org/data/transplant-trends/. Published 2019.

7. Organ Donation Statistics / Organ Donor. Organdonor.gov. https://www.organdonor.gov/statisticsstories/statistics.html. Published 2019.

8. Low J, Crawford K, Manias E, Williams A. Stressors and coping resources of Australian kidney transplant recipients related to medication taking: a qualitative study. J Clin Nurs. 2017;26(11-12):1495-1507.

9. Bedouch, P., Tessier, A., Baudrant, M., Labarere, J., Foroni, L., Calop, J., Bosson, J.-L. and Allenet, B. (2012), Computerized physician order entry system combined with on-ward pharmacist: analysis of pharmacists' interventions. Journal of Evaluation in Clinical Practice, 18: 911-918. https://doi.org/10.1111/j.1365-2753.2011.01704.x.

10. Jared Olson, Adam L Hersh, Jeffrey Sorensen, Jeffrey Zobell, Collin Anderson, Emily A Thorell, Intravenous Vancomycin Therapeutic Drug Monitoring in Children: Evaluation of a Pharmacy-Driven Protocol and Collaborative Practice Agreement, Journal of the Pediatric Infectious Diseases Society, Volume 9, Issue 3, July 2020, Pages 334-341, https://doi.org/10.1093/ipids/piz036

11. Fischer, Michael A., et al. "Pharmacy-Based Interventions to Reduce Primary Medication Nonadherence to

Cardiovascular Medications." Medical Care, vol. 52, no. 12, 2014, pp. 1050-1054. JSTOR, www.jstor.org/stable/26417864. Accessed 30 June 2021.

12. Susan M. Abughosh, Xin Wang, Omar Serna, Chris Henges, Santhi Masilamani, Ekere James Essien, Nancy Chung, and Marc Fleming. A Pharmacist Telephone Intervention to Identify Adherence Barriers and Improve Adherence Among Nonadherent Patients with Comorbid Hypertension and Diabetes in a Medicare Advantage Plan. Journal of Managed Care \& Specialty Pharmacy 2016 22:1, 63-73. 
Figure I. Tacrolimus Levels Pre-consult vs. Post-consult

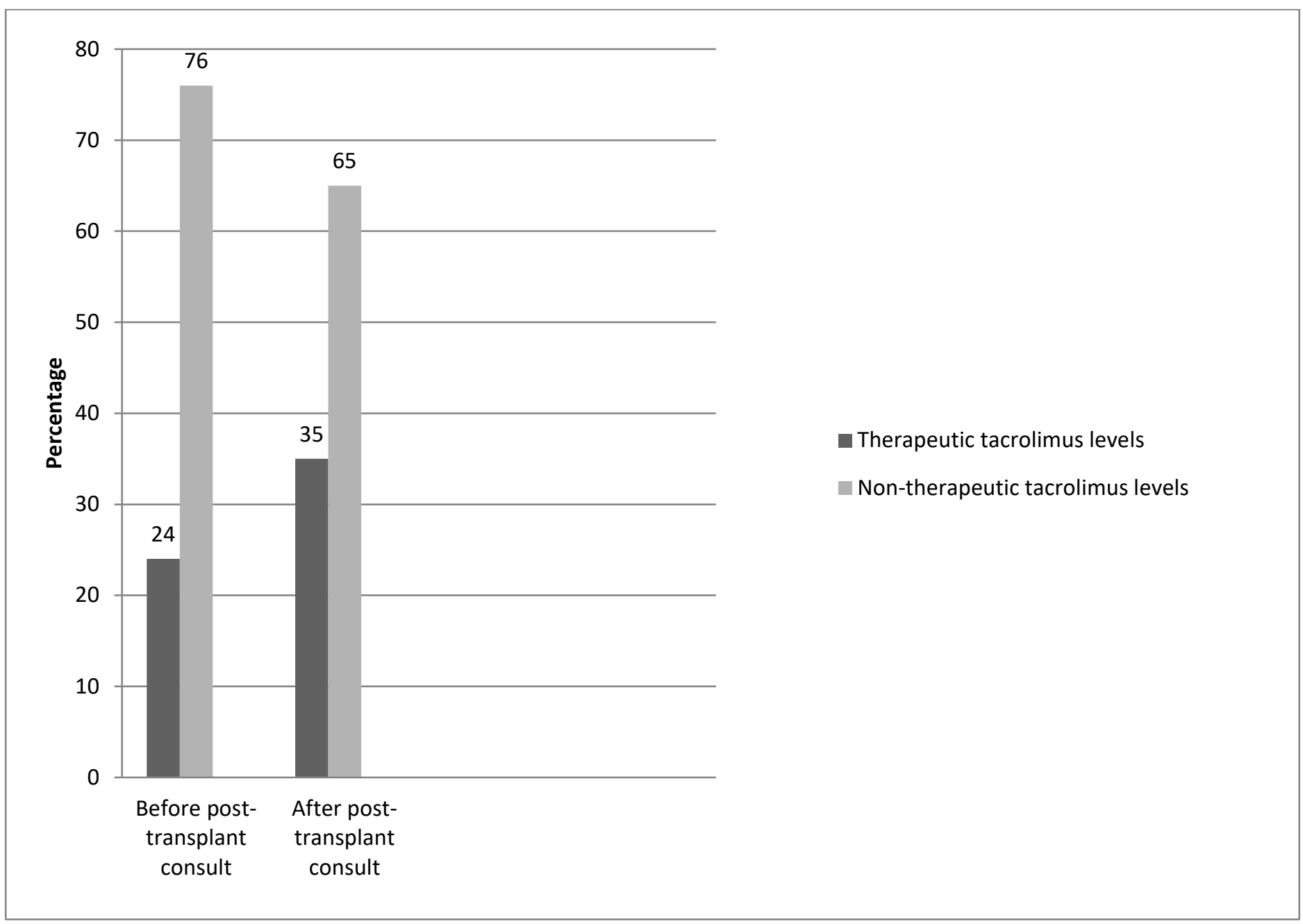

